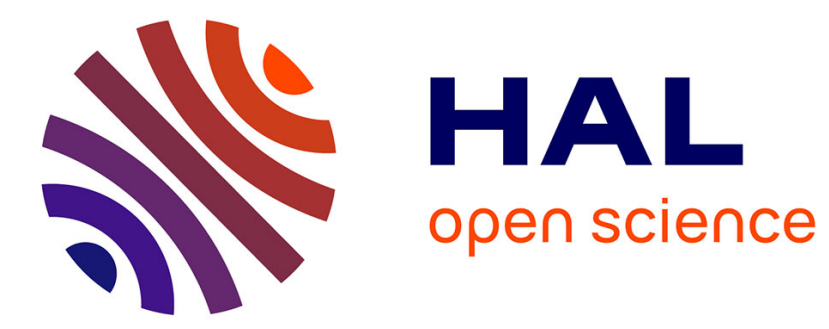

\title{
Registres émotionnels et processus politiques
}

Christophe Traïni

\section{To cite this version:}

Christophe Traïni. Registres émotionnels et processus politiques. Raisons politiques, 2017. hal02517808

\section{HAL Id: hal-02517808 \\ https://hal.science/hal-02517808}

Submitted on 24 Mar 2020

HAL is a multi-disciplinary open access archive for the deposit and dissemination of scientific research documents, whether they are published or not. The documents may come from teaching and research institutions in France or abroad, or from public or private research centers.
L'archive ouverte pluridisciplinaire HAL, est destinée au dépôt et à la diffusion de documents scientifiques de niveau recherche, publiés ou non, émanant des établissements d'enseignement et de recherche français ou étrangers, des laboratoires publics ou privés. 


\title{
Registres émotionnels et processus politiques
}

\author{
Christophe TRAÏNI \\ (IEP d'Aix-en-Provence, CHERPA)
}

Depuis quelques temps déjà, il n'est pas rare d'entendre dire que les sciences sociales, l'histoire, l'anthropologie, la sociologie et la science politique, seraient gagnées par un «emotional turn » ouvrant une nouvelle phase de leur histoire. Certes, une expression de ce type a le mérite d'inviter les chercheurs à se préoccuper de questionnements longtemps tenus pour anecdotiques et marginaux. Pourtant, elle présente également le risque de postuler une nouveauté radicale là où il s'agit, en fait, de répondre à des questions anciennes en articulant un peu mieux des ordres d'analyse jusqu'alors dispersés. Cette dispersion, comme chacun sait, doit souvent aux spécialisations qui tendent au cloisonnement des études relevant de disciplines telles la physiologie, la psychologie, les perspectives historiques et sociologiques, lesquelles se distinguent plus encore des approches normatives qui caractérisent la philosophie ou le droit. A cela s'ajoute le fait, qu'à l'intérieur de chaque discipline, les différents domaines d'étude peuvent s'avérer très inégalement enclins à s'intéresser aux dimensions émotionnelles de leur objet. A une échelle plus fine encore, et pour prendre l'exemple de l'étude des mobilisations, l'histoire des paradigmes qui ont marqué ce domaine rend parfois difficile la conjonction de ce qui relève de l'analyse des ressorts des engagements individuels, des opérateurs de coordination de l'action collective, et enfin des rapports aux institutions spécifiquement politiques. Or, l'un des principaux mérites de l'attention croissante accordée aux émotions réside précisément dans la manière dont elle nous invite à articuler ces trois types de questionnements qui tendent encore trop souvent à être traités de manière disjointe. Bien évidemment, il ne peut être question, pour autant, de s'en remettre aveuglement à l'idée d'une transdisciplinarité supposée ne présenter que des vertus. En matière de recherche, les logiques disciplinaires ne présentent pas que des désavantages pour peu que l'on saisisse bien les complémentarités de ce qui est prioritairement traité par les unes ou les autres. Ce dossier que Raisons politiques a décidé de consacrer aux émotions politiques constitue d'ailleurs l'occasion de montrer dans quelle mesure la thématique des émotions politiques permet de traiter de manière renouvelée un questionnement récurrent : à savoir celui des rapports entre, d'une part la philosophie et la théorie politique, et 
d'autre part les perspectives sociologiques. Bien sûr, d'innombrables travaux, à l'image de ceux de Luc Boltanski et Laurent Thévenot, ont déjà largement démontré l'utilité de développer des études au croisement de la philosophie et de la sociologie. De fait, il s'agira ici plus modestement de focaliser notre attention sur les réflexions soulevées par le chapitre introductif de ce dossier. Dans ce chapitre, Crystal Cordell mobilise les savoirs faires propres à la philosophie morale et à la théorie politique afin de caractériser les propriétés, aussi bien phénoménologiques et sociales, qui permettent de distinguer des états affectifs tels que le dégoût, l'indignation ou la pitié. Ce faisant, il s'agit bien là de mettre en exergue les logiques alternatives qui distinguent des registres émotionnels, c'est-à-dire des assortiments d'états affectifs interdépendants qui commandent des modes spécifiques de perception et de réaction à l'égard de certains objets et situations. Mon propos consistera donc à souligner en quoi de telles entreprises d'identification des propriétés des registres émotionnels sont particulièrement utiles à une sociologie des engagements et des mobilisations soucieuse d'interroger les rapports entre états affectifs et processus politiques. Loin de m'hasarder à discuter, en non spécialiste, du point de vue de la philosophie, je m'efforcerai plutôt de montrer dans quelle mesure l'approche théorique des émotions peut parfois s'enchâsser au cœur même des processus qui conduisent des individus à coordonner leur action en vue de faire valoir une cause collective ${ }^{1}$. Conformément à mon habitude, que certains pourraient assimiler à un indéfectible réflexe positiviste, je m'obligerai à étayer mon propos sur les observations empiriques que $\mathrm{j}$ ' ai pu relever auprès des promoteurs de la protection animale.

\section{Réactions affectives immédiates et évaluations intellectuelles}

C'est désormais un lieu commun de souligner que les apports récents des neurosciences ont largement contribué à renouveler notre manière d'envisager les rapports entre, d'une part les émotions, et d'autre part l'intelligence grâce à laquelle les organismes développés interagissent avec leur environnement. Contre le dualisme cartésien du corps et de l'esprit, de la passion et de la raison, l'étude des mécanismes du cerveau a révélé l'inextricable imbrication qui lie les états affectifs et les opérations mentales qui permettent aux êtres humains d'appréhender le monde qui les entoure ${ }^{2}$. Bien évidemment, le format et le propos de ce texte nous obligent ici à rappeler très sommairement quelques points essentiels des apports des neurosciences. En tout premier lieu, les émotions ne peuvent être dissociées d'une forme de «préséance du corps $^{3} »$. Par là, il faut entendre que la « peur », la « haine », «l'indignation », avant de pouvoir devenir des catégories cognitives et discursives ordonnant le monde des représentations et des discours, sont avant tout des altérations physiques du corps qui

\footnotetext{
${ }^{1}$ L'expression «cause » désigne ici la conviction qu'une action est nécessaire afin de réduire l'intolérable écart qui est perçu entre ce qui est et ce qui devrait être.

${ }^{2}$ Antonio Damasio, L'erreur de Descartes : La raison des émotions, Paris, Odile Jacob, 1995.

${ }^{3}$ Op. cit., p. 15.
} 
répondent à des discontinuités que le cerveau perçoit dans les flux d'information qui l'alimentent continument. Cette nature physiologique, réactive et transitoire, des phénomènes affectifs ne doit pourtant pas nous porter à ignorer les différentes formes de traitement que le cerveau réserve à ces signaux qui résultent de sources très hétérogènes. Dans certains cas, l'état affectif éprouvé relève simplement d'une conscience phénoménale qui se limite à attirer l'attention sur des faits qui appellent une réaction immédiate du corps : sursaut, tremblement, répulsion, fuite, etc... Dans d'autres cas, les mêmes états affectifs peuvent faire l'objet d'une conscience d'accès ou conscience réfléchie qui s'applique à soumettre les réactions corporelles à une évaluation mentale ${ }^{4}$. C'est bien cette évaluation d'ordre second qui permet non seulement l'entendement des émotions, c'est-à-dire leur compréhension pour ceux-là mêmes qui les éprouvent, mais encore leur inhibition ou intensification en fonction de leur nature jugée adéquate ou non à la situation. Bien plus encore, sans cette évaluation d'ordre second, les états affectifs ne pourraient pas être clairement exprimés et ouvrir la voie à une compréhension intersubjective, voire à une véritable coordination de l'action (dans la mesure où les émotions manifestées par autrui affectent souvent le cours de l'action de soi). Autant dire que nos modes d'agir et d'interagir dépendent d'un inextricable entremêlement de différents types d'états affectifs que l'on peine parfois à bien distinguer analytiquement.

Ainsi, et en tout premier lieu, certaines formes d'engagement dans l'action pourraient être qualifiées — si l'on nous permet ce mauvais jeu de mots d'engagement par corps et par cœur. Des engagements par corps, dans la mesure où ils se manifestent par des réactions perçues comme immédiates et inéluctables à l'instar de la peur, du dégoût, ou bien encore, dans un tout autre registre, de la pitié que suscite le spectacle de la souffrance du malheureux. Bien évidemment, ces engagements par corps opèrent également par cœur au sens où leur automaticité dépend de l'activation d'une mémoire psychomotrice ou plus exactement d'apprentissages si bien assimilés que leur caractère laborieux passé a fini par disparaître de la conscience réfléchie. En d'autres termes, certaines émotions s'imposent à nous sur un mode d'autant plus impérieux qu'elles résultent des sensibilités forgées par l'accumulation d'expériences affectives tout au long de notre socialisation préalable. A l'autre extrémité du continuum de nos manières de réagir aux évènements qui nous entourent se trouvent des émotions bien plus dépendantes des modalités réflexives de nos activités cérébrales. Dans la pensée occidentale, cette faculté a donné lieu à une métaphore oculaire qui nous porte à imaginer que notre pensée opère sur des représentations intérieures : «notre esprit est en quelque sorte un miroir dans lequel la réalité se reflète; nous possédons un œil interne qui nous permet de regarder dans ce miroir et d'inspecter les images qui s'y présentent. Nous nous livrons sur ce miroir au travail de la réflexion que Vauvenarges définit comme la "puissance de se replier sur ses idées, de les examiner, de les modifier ou de

\footnotetext{
${ }^{4}$ Pierre Buser, L'inconscient aux mille visages, Paris, Odile Jacob, 2005, p. 108.
} 
les combiner" ${ }^{\prime} \gg$. En matière d'émotion, c'est bien cette modalité réflexive de notre activité cérébrale qui permet de distinguer des réactions affectives, tel le dégoût, la peur ou la colère, d'émotions morales telle l'indignation. Cette dernière, en effet, loin de simplement impliquer l'activation d'une répulsion, suppose l'élaboration d'un jugement moral permettant d'établir que le comportement d'un tiers transgresse celui qui devrait être, en général, attendu. De telles évaluations intellectuelles impliquent donc une tendance à l'abstraction, c'est-à-dire à la focalisation de l'attention, non plus seulement sur les contingences transitoires du monde sensible, mais sur des essences, des concepts et des principes jugés prépondérants. De fait, comme le montre bien le texte de Crystal Cordell, à partir des réflexions de philosophes tels Aristote ou Adam Smith, les réactions affectives des êtres humains peuvent faire l'objet d'analyse visant à établir les principes d'ordre général qui permettent de conclure à leur justesse et à leur adéquation à la justice. Ainsi, l'indignation ne se résume pas à une simple réaction physiologique de haut-le cœur ou de colère, elle repose sur le travail d'une conscience réfléchie qui pointe vers des représentations abstraites de ce qu'il est juste d'éprouver et de manifester. Bien plus encore, dans la perspective des sociologues, cette évaluation intellectuelle, qui pour le neurologue ressort d'une conscience d'accès, se manifeste sous des modalités diverses. Le plus souvent, cette évaluation morale relève d'une « conscience pratique » à la portée de tous ceux qui sont en mesure de faire preuve des compétences nécessaires pour s'adapter au monde au sein duquel ils ont été socialisés ${ }^{6}$. Comme le suggère la contribution de Paula Vasquez Lezama dans ce dossier, en se déployant sur le mode de la protestation, cette conscience pratique de l'indignation peut également adopter le «registre de la dénonciation de l'intolérable », s'efforçant ainsi de défier le régime émotionnel dominant. Il arrive parfois, cependant, que cette évaluation morale des réactions affectives implique une «conscience discursive » qui distingue ceux qui - à l'image des philosophes — entendent se dédier à l'analyse et à la discussion des principes généraux d'une éthique visant à orienter les conduites morales des êtres humains ${ }^{7}$.

Ainsi, l'examen des propriétés respectives de la pitié, du dégoût ou de l'indignation présente le mérite de nous inviter à bien saisir ce qui distingue, d'une part les réactions affectives qui se suffisent en elles-mêmes, d'autre part des émotions morales, c'est-à-dire qui impliquent un travail de justification pointant vers

\footnotetext{
${ }^{5}$ Jean-François Billeter, «Pensée occidentale et pensée chinoise : le regard et l'acte », dans Différences, valeurs, hiérarchie, Textes offerts à Louis Dumont, réunis par Jean-Claude Galey, Paris, EHESS, 1984, p. 31.

${ }^{6}$ Rappelons ici que, selon Anthony Giddens, la conscience pratique désigne «tout ce que les acteurs savent (ou croient) des conditions sociales, en particulier ce qu'ils savent des conditions de leur propre action, mais qu'ils ne peuvent exprimer de façon discursive », Anthony Giddens, La constitution de la société, Paris, P.U.F, 1987, p. 440.

${ }^{7}$ La conscience discursive, pour sa part, désigne «tout ce que les acteurs peuvent exprimer de façon verbale (orale ou écrite) des conditions sociales, en particulier celles de leur propre action. Une conscience [awareness] qui prend une forme discursive », Ibid ibidem.
} 
l'identification de principes supérieurs communs ${ }^{8}$. Bien plus encore, en faisant appel aux analyses sophistiquées des maîtres de la philosophie, ce type d'étude nous rappelle que cette moralisation des émotions peut devenir une forme d'activité très spécialisée qui demandent des appétences et des compétences discursives qui sont loin d'être les plus couramment partagées au sein du monde social.

Loin d'être une fin en soi, ces distinctions ne visent ici qu'à élucider dans quelle mesure différentes formes d'états affectifs peuvent étayer les processus d'engagement en faveur d'une cause, en l'occurrence, et pour l'exemple, la protection animale. Ce terrain d'enquête, en effet, présente une grande diversité de modes d'action et de profils militants qui attestent de la pluralité des registres émotionnels susceptibles de soutenir ceux qui se sont promis de se dédier à la cause. De ce point de vue, un premier contraste s'impose d'emblée en ce qui concerne deux manières, parfois distinctes, parfois complémentaires, de s'émouvoir des mauvais traitements infligés aux animaux. Pour un bon nombre d'activistes, prendre part à la protection des animaux revient à s'engager dans des actions qui permettent, non seulement de répondre aux sentiments que les souffrances des bêtes éveillent en eux, mais encore d'éprouver d'autres émotions jugées attrayantes ou nécessaires : usage de caméras cachées visant à dévoiler l'horreur des abattoirs, opérations commando lancées afin de délivrer les cobayes destinés à l'expérimentation scientifique, et bien plus souvent encore soins et nourriture apportés à des bêtes sauvages blessées ou des chiens et des chats abandonnés... Ici, la volonté d'apaiser au plus vite les tourments des êtres vulnérables, ou bien encore d'entraver coûte que coûte les pratiques de leurs tortionnaires, prime sur tout autre considération. A l'autre extrémité du continuum des pratiques d'engagement se trouvent plutôt des protecteurs des animaux dont les activités s'apparentent bien plus aux formes de militantisme auxquelles la science politique a l'habitude de s'intéresser. Cette minorité, en effet, loin de se contenter de vouloir remédier à la souffrance animale, y compris en invitant leur concitoyens à les soutenir dans leur entreprise, manifeste une forte propension à envisager leur pratique comme l'engagement dans un combat d'idées et d'arguments. Ici, il s'agit autant de valoriser des compétences cognitives et discursives que des appétences puisque ces activistes-là doivent apprendre à apprécier les excitations spécifiques qui résultent de l'engagement dans les raisonnements abstraits de l'éthique, et plus encore, dans des jeux interlocutoires d'échanges d'arguments inextricablement liées à des états affectifs de type agonistique ${ }^{9}$. Cette tendance est particulièrement nette auprès de ceux qui se réclament de la défense des droits des animaux, à l'image des membres de la Ligue Française des Droits des Animaux, ou bien encore des promoteurs de l'antispécisme ; autrement dit des thèses défendues par des

\footnotetext{
${ }^{8}$ Luc Boltanski, Laurent Thévenot, De la justification. Les économies de la grandeur, Gallimard, 1991.

${ }^{9}$ Rappelons, une fois de plus, l'importance de cette distinction à travers laquelle Bernard Lahire nous invite à bien distinguer, d'une part la compétence ou capacité à faire telle ou telle chose, et d'autre part l'appétence ou le goût et l'envie de la faire ; Bernard Lahire, « De la théorie de l'habitus à une sociologie psychologique », in Bernard Lahire, Le travail sociologique de Pierre Bourdieu, Paris, La Découverte, 2001, p. 121-152.
} 
auteurs qui, à partir de la seconde moitié des années 1970, développent au sein des universités anglophones une éthique animale conçue comme un domaine spécialisé de la philosophie morale ${ }^{10}$. Pour ces protecteurs des animaux là, se dédier à la cause animale implique de se préoccuper, non seulement de la souffrance des bêtes, mais encore d'être en mesure de saisir les raisonnements développés par des auteurs tels Peter Singer, Tom Regan, Gary Lawrence Francione, auxquels peuvent s'ajouter des précurseurs tels Théophraste ou Jeremy Bentham. Ici, on le voit, l'engagement se fait combat d'idées et s'applique à s'armer d'une théorie politique des émotions en mesure de (dis)qualifier et donc de moraliser - les états affectifs que les hommes associent généralement aux interactions avec les animaux : le spécisme, par exemple, est défini comme «l'idéologie qui justifie et impose l'exploitation et l'utilisation des animaux par les humains de manières qui ne seraient pas acceptées si les victimes étaient humaines ${ }^{11}{ }^{11}$. Ainsi, on le voit, l'approche théorique des émotions - relative à la manière dont les hommes traitent les bêtes - fraye parfois la voie à un registre émotionnel spécifique qui ne constitue qu'une option parmi d'autres dès lors que des militants entendent se dédier à la protection des animaux.

\section{Des régimes émotionnels variés afin de mieux (se) convaincre}

Bien évidemment, l'intérêt heuristique que la sociologie des mobilisations peut trouver dans l'attention récente pour les émotions ne se résume pas seulement à cette distinction idéaltypique entre, d'une part les engagements par corps, et d'autre part l'intellectualisation de sensibilités agonistiques. Bien d'autres contrastes et nuances permettent de distinguer des variations de registres émotionnels que l'observateur ne peut ignorer s'il veut pouvoir rendre compte de la diversité des manières dont des entrepreneurs de mobilisation s'efforcent de faire valoir, à leurs propres yeux comme à ceux d'autrui, l'importance de leur cause. Dans cette optique, et afin de déjouer le soupçon d'impressionnisme, la notion de dispositifs de sensibilisation permet d'étayer l'analyse sur un matériel empirique bien tangible puisqu'il s'agit de désigner l'ensemble des supports matériels, des agencements d'objets, des mises en scènes, que les militants déploient afin de susciter des réactions affectives qui prédisposent ceux qui les éprouvent à s'engager ou à soutenir la cause défendue. La notion invite donc à interroger, dans une perspective pragmatique, la manière dont des entrepreneurs de cause s'appliquent à exprimer des émotions tout en sollicitant celles des publics qui les entourent. La description quasi-ethnographique qu'elle présuppose conduit surtout l'observateur à saisir les logiques respectives des divers registres émotionnels que les dispositifs de sensibilisation alimentent.

\footnotetext{
${ }^{10}$ On trouvera une analyse des différentes positions et des débats philosophiques contemporains en éthique animale dans Jean-Baptiste Jeangène Vilmer, Ethique animale, Paris, Presses Universitaires de France, collection "Ethique et philosophie morale", 2008.

${ }^{11}$ Définition http://www.cahiers-antispecistes.org/spip.php?article13. Consulté le 13 janvier 2015.
} 
Ainsi, dès lors qu'il s'agit de dénoncer l'écart intolérable entre les traitements réservés aux animaux et ce qu'ils devraient être, les protecteurs des animaux peuvent avoir recours à des dispositifs qui invitent à éprouver des émotions de nature très variable. En d'autres termes, pour les protecteurs des animaux, le dégoût et l'indignation constituent les pôles d'un continuum à l'intérieur duquel prennent place les différentes manières dont ils peuvent s'émouvoir de manière congruente à leur engagement.

Schéma 1. Des dispositifs visant à dénoncer les mauvais traitements infligés aux animaux

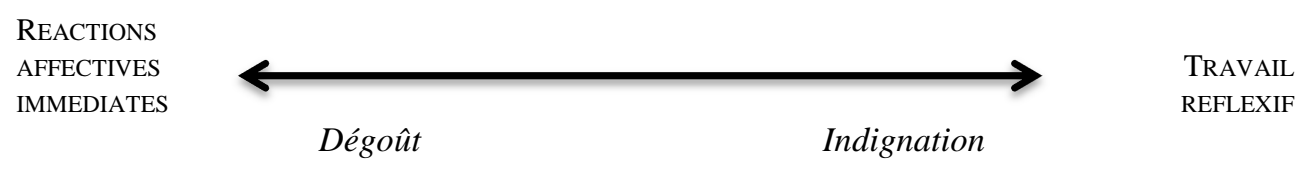

\begin{tabular}{|c|l|c|c|}
\hline Mode FIGURATIF & Représentations artistiques & Dialogues imaginaires & Argumentations philosophiques \\
\hline MODE REALISTE & Comptes rendus réalistes & Témoignages & Exposés techniques \\
\hline
\end{tabular}

Dans certains cas, des descriptions les plus réalistes possibles — et mieux encore des photos ou des enregistrements audiovisuels — rendent compte des scènes les plus abjectes et horrifiantes des pratiques ayant cours dans les laboratoires, les arènes de la corrida, les abattoirs, etc... ${ }^{12}$. Ces descriptions, qui se veulent immédiates, aussi bien dans leur mode de restitution que dans le type de réactions affectives qu'elles sollicitent, se distinguent de celles qui ont recours à la figure du témoignage, c'est-à-dire à une forme de narration d'autant plus émouvante qu'elle intègre à son compte rendu la description des réactions affectives qui ont étreint celui qui a été le témoin de scènes horrifiantes ${ }^{13}$. L'histoire de la protection animale a ainsi donné lieu à de multiples récits de sympathisants de la cause racontant l'épreuve affective qu'a constituée, pour eux, la découverte des souffrances effroyables infligées aux animaux. Ici, la mise en exergue des émotions éprouvées par le témoin s'intercale entre l'horreur crue de la souffrance animale et le public invité à réagir au scandale dénoncé. Parfois, d'autres dispositifs de sensibilisation s'appliquent plus encore à étayer l'indignation sur la capacité d'imagination, d'abstraction et de raisonnement analogique du public. Nous pensons ici aux nombreuses pratiques artistiques auxquelles les protecteurs des animaux ont souvent recours à l'image de ces tableaux, dessins, poèmes ou chansons. De manière significative, de tels dispositifs de sensibilisation s'éloignent du simple dégoût viscéral en lui associant des formes d'appréciation esthétique qui persuadent les sympathisants

\footnotetext{
12 En mars 2016, l'actualité politique française a été marquée par le recours à de tels procédés : l'émoi suscité par la diffusion des images des abattoirs d'Alès et du Vigan, enregistrées par l'association L-214 Éthique \& Animal, conduit à la mise en place d'une commission d'enquête parlementaire. Suite à une nouvelle vidéo concernant un autre abattoir, le ministre de l'agriculture annonce des inspections spécifiques sur la protection animale dans tous les abattoirs de France avant la fin du mois d'avril.

${ }^{13}$ Dominique Mehl, «Le témoin, figure emblématique de l'espace privé/public », dans Daniel Cefaï, Dominique Pasquier (dir.), Les sens du public. Publics politiques, publics médiatiques, Paris, PUF, 2003, p. 489-502.
} 
de la cause de la nécessité d'éprouver une juste colère. Dans cette optique, les dispositifs de sensibilisation incitent le plus grand nombre à réagir affectivement, par exemple, à des dessins stylisés des calvaires des victimes animales jouxtant des poèmes s'adressant directement à eux. L'émotion est à son comble lorsque l'animal martyrisé est lui-même le narrateur du poème invitant les hommes à un dialogue imaginaire des plus poignants à l'instar, par exemple, de ce chien viverrin exploité pour sa fourrure : «je n'ai plus d'oreilles, je n'ai plus de nez / Des larmes de sang coulent de mes yeux / J'ai tellement de mal à respirer / Mon âme entière vous supplie mon Dieu / On m'a battu et on m'a frappé / Maintenant que je suis mort vivant / Accroché, je me suis réveillé / la peau arrachée, le corps en sang ${ }^{14} \gg$. Bien évidemment, il n'est pas rare que le chant, et les tonalités affectives dramatiques des musiques d'accompagnement permettent plus facilement encore l'accès à ce registre émotionnel qui combine représentation figurative et capacité à imaginer des dialogues bouleversants avec ces animaux que l'on se propose de secourir.

Cependant, certains dispositifs de sensibilisation alimentent un autre type de régime émotionnel qui se distingue en faisant plus encore appel à des capacités d'abstraction et de mises à distance des réactions affectives immédiates. Du fait des compétences scolaires et intellectuelles qu'ils requièrent, ces derniers s'apparentent à des dispositifs experts, c'est-à-dire des agencements d'objets, des mises en scènes et des discours faisant valoir une maîtrise de connaissances échappant au profane. Dans certains cas, ces dispositifs visant à dénoncer — selon leur mode spécifique — le sort abominable que les hommes réservent indûment aux bêtes se présentent sous la forme d'exposés didactiques à tonalité souvent technique. A titre d'exemple, on pourrait évoquer ici la brochure «Découvrez la corrida» produite par le CRAC, le Comité Radicalement Anti-Corrida. Pour chacun des 3 actes (tercios) de la corrida, la brochure présente une présentation en coupe de l'anatomie du taureau laissant voir son squelette tandis que les commentaires pourraient faire penser à une autopsie médicale : «la blessure atteint parfois les nerfs rachidiens à leur sortie des vertèbres »; le matador s'applique à enfoncer « une épée dans la cage thoracique, couper un gros vaisseau près du cœur et provoquer une hémorragie interne »; «le puntillero plonge son arme juste derrière le crâne, pour atteindre le bulbe rachidien »... S'il est bien toujours question de susciter le dégoût, il est clair toutefois que les réactions affectives sollicitées ici ne sont pas de même nature que celles que provoquent, par exemple, les photographies de chevaux de picador éventrés par les cornes d'un taureau. Les graphiques et les énoncés experts, en effet, étayent l'indignation morale en combinant répugnance pour la pratique et cette forme spécifique d'excitation qui résulte de la possibilité d'exciper d'une connaissance qui dévoile ce que des individus malveillants prétendent occulter. Si dans ce cas ce sont les connaissances anatomiques qui paraissent appropriées, il n'est pas rare que certains protecteurs des animaux aient recours aux analyses de la philosophie afin

\footnotetext{
14 «Parole d'un chien viverrin. Histoire vraie », http://animauzine.net/parole-d-un-chien-viverrin.html, consulté le 12/04/2016.
} 
de requalifier, à travers des catégories et des principes abstraits, les ressorts justifiant la répugnance et la désapprobation devant s'abattre sur le sort réservé aux animaux. Nous pouvons évoquer ici l'exemple de la promotion de l'antispécisme, rencontré plus haut, qui consiste à faire valoir qu'à travers l'opposition aux multiples formes d'exploitation des animaux, il s'agit, en fait, de lutter bien plus largement contre des injustices que l'analyse éthique est la mieux à même de débusquer : «le spécisme est à l'espèce ce que le racisme et le sexisme sont respectivement à la race et au sexe ; la volonté de ne pas prendre en compte (ou de moins prendre en compte) les intérêts de certains au bénéfice d'autres, en prétextant des différences réelles ou imaginaires mais toujours dépourvues de lien logique avec ce qu'elles sont censées justifier ${ }^{15}$ ». Ici, la nature experte de cette manière de procéder découle de l'intertextualité des énoncés qui s'appliquent à édifier un corpus de textes de référence et à travers laquelle s'éprouve cette forme d'excitation propre à l'érudition et au «combat des idées »: exhumer les propositions oubliées d'illustres précurseurs tels Plutarque ou Bentham; convoquer les références faisant autorité à l'image de Peter Singer ou Thomas Regan; savoir reconnaître une argumentation déduite des principes utilitaristes (et pourquoi pas proposer des prémisses alternatives à l'instar de l'éco-féminisme ?) ; voire pour les plus experts distinguer perspectives déontologique ou conséquentialiste...

On prendra soin de noter que repérer ainsi les logiques propres aux multiples registres émotionnels est d'autant plus utile que ces derniers présentent souvent de très fortes affinités avec les différents profils militants qui structurent l'espace des organisations et des mobilisations. Dans le cas de la protection animale, aujourd'hui en France, la plus grande part des adeptes se dédient corps et âme à la cause en mettant en œuvre des pratiques qui laissent bien peu de place au souci de produire les meilleurs arguments destinés à des joutes verbales jugées primordiales. D'autres protecteurs des animaux peuvent, au contraire, manifester le souci de s'engager dans des combats d'arguments. S'ils constituent une minorité sur le terrain de la protection animale, les profils militants qui leurs sont analogues apparaissent souvent majoritaires dans le cas des causes qui préoccupent plus aisément la science politique (partis, syndicats, mouvements altermondialistes, organisations environnementales, etc...). Dans de tels types de cas, les dimensions affectives des engagements résultent du fait qu'ils nécessitent une certaine appétence pour les excitations de type agonistique, et plus particulièrement pour l'affirmation d'idées invitant sympathisants et adversaires de la cause à se positionner.

\section{L'impact politique des régimes émotionnels}

Ainsi, les contrastes qui caractérisent l'engagement en faveur de la protection animale présentent un enseignement précieux pour l'étude des dimensions

\footnotetext{
15 Les Cahiers antispécistes. Réflexion et action pour l'égalité animale http://www.cahiers-
} antispecistes.org/spip.php?article13 Consulté le 19 avril 2016. 
émotionnelles des processus de mobilisation visant à remédier à un état du monde jugé insatisfaisant. En définitive, le prosélytisme discursif n'a rien d'inéluctable et les chercheurs devraient, à l'encontre de tout biais intellectualisant, pouvoir traiter de manière symétrique des entrepreneurs de cause qui peuvent faire preuve d'une propension très inégale à la production de discours visant à justifier leurs propres conduites. Dans le présent dossier, l'étude de Paula Vasquez Lezama permet de mettre au jour le registre «hybride » d'un gréviste de la faim, entre émotion et expertise, qui « revendique ses droits en mobilisant un discours savant et expert à partir d'une performance corporelle sensationnelle et spectaculaire ». Par prosélytisme discursif, il faut entendre ici l'ensemble des pratiques qui s'efforcent de convertir autrui à ses convictions à travers des discours, c'est-à-dire l'énonciation d'arguments se présentant comme les conséquences du raisonnement et de la justification ${ }^{16}$. Le fait que cette forme de mise à l'épreuve des émotions ne constitue qu'une alternative parmi d'autres nous invite, bien évidemment, à interroger ses effets spécifiques sur le devenir des processus de mobilisation collective. En d'autres termes, la valorisation du prosélytisme discursif tend-elle à favoriser ou défavoriser la probabilité qu'une action collective d'envergure se déploie autour d'une préoccupation commune ? Là encore, la diversité des formes d'engagement qui caractérise la protection animale permet d'apporter une réponse équilibrée à un tel questionnement.

En tout premier lieu, l'histoire de la protection animale offre plusieurs occasions d'examiner ce que la convergence de ses sympathisants autour d'une indignation morale - en apparence commune - doit ou non à la solidité d'une argumentation reposant sur des principes explicites et univoques. L'histoire très significative, longue et ancienne, des émotions fomentées par les promoteurs de l'opposition à la vivisection est évidemment trop complexe pour pouvoir être évoquée ici $^{17}$. Heureusement, l'exemple de mobilisations plus récentes contre la corrida s'avérera sans doute suffisant pour mettre en exergue la part très relative des justifications discursives dans la mise à l'épreuve d'une indignation en mesure d'assurer la convergence de groupes de soutien très hétérogènes. De ce point de vue, l'observation participante de manifestations de rue rassemblant des opposants à la tauromachie est riche d'enseignements ${ }^{18}$. A première vue, les individus prenant part à ces défilés semblent d'autant plus partager une émotion commune que la manifestation est l'occasion de manier et brandir des dispositifs de sensibilisation qui relèvent d'un registre émotionnel du dévoilement oscillant entre un mode réaliste ou figuratif : photos abjectes de taureaux mortellement blessés, tambours

\footnotetext{
${ }^{16}$ Dans une perspective pragmatique, la justification suppose l'aptitude à rapporter des conduites à des principes généraux dont la validité dépasse les circonstances singulières de leur énonciation.

${ }_{17}$ Christophe Traïni, La cause animale (1820-1980). Essai de sociologie historique, Paris, Presses Universitaires de France, 2011 ; "Opposing Scientific Cruelty: The Emotions and Sensitivities of Protestors against Experiments on Animals", Contemporary European History, volume 23/04, 2014, p. 523-543.

${ }^{18}$ Christophe Traïni, « Dramaturgie des émotions, traces des sensibilités. Observer et comprendre des manifestations anti-corrida », ethnographiques.org, $\mathrm{n}^{\circ} 21$, novembre 2010
} 
funéraires, sifflets stridents utilisés comme pour lancer une alerte, déguisements évoquant métaphoriquement les victimes de tortures, manifestants maquillés de larmes de sang, etc... Pourtant, les entretiens réalisés auprès de militants, mais encore un certain nombre d'indices collectés aux cours de l'enquête, permettent de percevoir à quel point l'indignation publiquement exprimée prolongent, en fait, des sensibilités très hétérogènes que les uns et les autres doivent à leur histoire familiale, affective et sociale. Enseignants du secondaire, chrétiens protestants (parfois même évangélistes), philosophes spécialistes des questions morales, femmes tenant des refuges pour chiens, admiratrices de Brigitte Bardot, anarchistes de Charlie-Hebdo: tout indique que l'expression de l'indignation à l'égard de la corrida autorise des formes d'accord infraargumentatif entre des individus qui auraient bien du mal à s'entendre s'ils devaient expliciter discursivement les principes ultimes justifiant leur ralliement à la cause. Ceci est d'autant plus vrai que, répétons-le, les opposants à la corrida font très inégalement preuve de cette propension à envisager l'engagement comme un combat d'idées et d'arguments. Ainsi, par exemple, pour les bénévoles du refuge de la Société Protectrice des Animaux de Marseille, qui avait affrété un autocar en direction de la manifestation à Nîmes en septembre 2008, l'indignation à l'encontre de la tauromachie, manifestée à travers leur participation au défilé, prolonge tout naturellement cette pratique régulière consistant à prendre soin des bêtes abandonnées ou maltraitées par les hommes. A cela s'ajoute encore le fait que, comme le révèlent certains entretiens avec les protecteurs des animaux, l'indignation exprimée à travers ces performances collectives ne peut être dissociée des sensibilités personnelles liées à des expériences affectives qui relèvent d'une intimité peu encline à la publicisation: violence psychologique ou physique exercée par les parents, confrontation à des discriminations raciales, volonté de s'affirmer en dépit des assignations de genre, situation professionnelle valorisant bien peu des dispositions intellectuelles et critiques acquises au cours des études, etc...

Autant dire qu'une bonne compréhension de la complexité des états affectifs liés à des engagements militants nous invite à récuser l'idée que la coordination de l'action collective à l'origine des mobilisations implique nécessairement une homogénéité des représentations mentales et des sensibilités éprouvées. Dans cette optique, il convient de se méfier des modes de description qui tendent à faire appel à la figure du «partage des émotions ». Le fait que de multiples individus puissent être collectivement engagés dans l'expression d'une émotion apparemment commune ne doit pas nous porter à perdre de vue les formes plurielles de sensibilités qui les distinguent : «c'est le plus souvent sur la base de représentations, projets, conceptions du juste et du souhaitable, intérêts disparates (et en outre susceptibles d'évoluer) que des agents ou des groupes rejoignent une "même" mobilisation et contribuent, ce faisant, à lui conférer sa stature collective $^{19}$ ». De fait, on perçoit bien ici à la fois l'intérêt et les limites d'une approche

\footnotetext{
${ }^{19}$ Lilian Mathieu, L'espace des mouvements sociaux, Éditions du Croquant, 2012, p. 259. Bien évidemment, cette nature intrinsèquement composite des mobilisations peut s'avérer plus ou moins marquées en fonction des cas.
} 
exclusivement focalisée sur les justifications discursives (et sur les théories des émotions qui en résultent). Un intérêt certain dans la mesure où cette approche met bien en exergue les efforts de rationalisation discursive et d'homogénéisation à travers lesquels les élites des mobilisations s'appliquent à définir les principes supérieurs que le plus grand nombre devrait reconnaître. Quant aux limites de cette approche, elles découlent du fait que, comme on a pu le voir, cette préoccupation est loin de pouvoir rendre compte des ressorts de l'engagement de tous ceux qui convergent autour d'une expression collective d'indignation. Pour le dire autrement, « la production de discours, et spécialement de rationalisation et de justification de l'engagement protestataires, fait partie intégrante de la pratique des mouvements sociaux. [Toutefois], cette production discursive n'est pas un préalable de la mobilisation mais une de ses composantes, et son adoption par ceux qui y participent n'est pas une condition de leur engagement mais un des aboutissements possibles ${ }^{20} \gg$.

On aura bien sûr compris que la perspective adoptée ici ne vise rien d'autre qu'une meilleure compréhension des procédures infra-argumentatives qui favorisent le développement d'une mobilisation en faveur d'une cause collective. Elle ne doit en aucun cas nous porter à conclure que les productions argumentatives et théoriques des militants n'ont aucune incidence sur le devenir politique de la cause qu'ils défendent. A vrai dire, il est impossible d'analyser les rapports entre émotions et politique en ignorant le fait que les institutions démocratiques font peser sur les citoyens porteurs de revendications des exigences de contrôle des affects, mais encore de maitrise des normes qui font apparaître la politique comme une confrontation d'idées et d'arguments. S'il convient de ne pas surestimer le rôle de la rhétorique dans les processus à l'origine des mobilisations collectives, il est tout aussi important de ne pas perdre de vue que, comme l'écrit Crystal Cordell, cette pratique discursive, perméable aux émotions, constitue depuis très longtemps un «art de la persuasion qui est éminemment publique et politique ». De fait, il n'est pas étonnant de constater que les protecteurs des animaux que les autorités publiques reconnaissent le plus aisément comme des interlocuteurs légitimes se distinguent par leur capacité à faire valoir que leurs propension à s'indigner de la maltraitance des animaux découle entièrement de la solidité de leurs analyses théoriques et philosophiques.

De ce point de vue, l'un des exemples les plus remarquables est sans aucun doute celui de La Fondation Droit Animal, Ethique et Sciences (LFDA). Alors même qu'elle aurait bien du mal à exciper un nombre de sympathisants supérieur à d'autres organisations dédiées à la cause, la LFDA est représentée au sein de plusieurs commissions ou comités consultatifs de ministères tels l'agriculture, l'écologie ou bien encore la recherche et l'enseignement supérieur. Cette légitimité auprès des instances de la décision politique ne résulte pas exclusivement de la maitrise du langage juridique et de la technicité des dossiers produits par la fondation. Ce qui distingue la LFDA des

\footnotetext{
${ }^{20}$ Lilian Mathieu, op. cit., p. 261.
} 
autres organisations de la protection animale, c'est bien plus encore sa capacité à invoquer une indignation raisonnable et raisonnée. Une indignation raisonnable qui, aux yeux des autorités publiques, est bien préférable à celles de militants bien plus radicaux, porteurs de revendications telles l'abolition de la viande ou des expérimentations animales, et de ce fait bien peu enclins aux discussions et aux compromis avec les professionnels des secteurs de l'agro-alimentaire ou de la recherche. Une indignation raisonnée, ensuite, qui distingue tout autant les membres de la LFDA de ces associations drainant d'innombrables sympathisants ne pouvant se réclamer que de leur passion pour les bêtes aimantes et souffrantes. Une part non négligeable des activités de la Fondation Droit Animal, Ethique et Sciences, en effet, conduit à établir une différence notable entre, d'une part de simples élans affectifs de dégoût ou de pitié, et d'autre part des émotions d'autant plus morales et dignes qu'elles auront fait l'objet de réflexions et de discussions mobilisant les compétences de la science, de la philosophie et du droit. Ainsi, la LFDA organise régulièrement des conférences et des colloques aux intitulés éloquents : «Humanité, animalité: quelles frontières philosophiques ?», «Pensée et conscience chez l'animal », «En morale et en esthétique, sommes-nous des philosophes ou des singes ? ; «Le respect de l'animal dans ses racines historiques : de l'animal-objet à l'animal sensible », etc... Ces colloques et conférences ont le plus souvent donné lieu à la publication d'ouvrages et de brochures présentant les argumentations de philosophes, magistrats, médecins, biologistes, paléontologues, vétérinaires... De son côté, Georges Chapouthier, biologiste et philosophe, viceprésident de la LFDA développe dans son ouvrage, Kant et chimpanzé. Essai sur l'être humain, la morale et l'art, publié chez Belin en 2009, un argument récurrent des membres de la Fondation. Comme dans d'autres organisations de la protection animale, il est bien question de promouvoir la pitié à l'égard des bêtes mais en se préoccupant ici de la justifier à partir de connaissances se réclamant de la philosophie, des neurosciences, des sciences biologiques et médicales. Le propos consiste, en effet, à relever le fait que les évolutions récentes dans ces divers domaines de la science nous invitent à conclure que les animaux éprouvent eux aussi des émotions. De fait, cette continuité des propriétés affectives entre les animaux et les hommes dicte à ces derniers de développer à l'égard des premiers des sentiments de pitié relevant d'une morale fondée sur les raisonnements de la science et de la philosophie. En d'autres termes, c'est bien une théorie des émotions (des animaux et des hommes) qui est forgée ici, non seulement pour protester contre le sort des bêtes, mais encore pour améliorer la dignité morale des hommes tout en orientant l'évolution des règlements juridiques et des politiques publiques à travers les nombreuses expertises que la LFDA destine aux décideurs politiques. Une belle illustration de la manière dont l'approche théorique des émotions peut étroitement s'imbriquer à des processus politiques au cours desquels l'engagement de militants passionnés et les procédures codifiées d'une bureaucratie légale-rationnelle ne cessent d'interagir. 